\title{
TRAINING OF AEROBIC AND ANAEROBIC FITNESS IN CHILDREN WITH ASTHMA
}

\author{
Françols-Pierre Counil, MD, PhD, Alain Varray, PhD, Stephan Matecki, MD, PhD, Alain Beurey, MD, Patrick Marchal, MD, \\ Michel VOISIN, MD, AND ChRISTIAN PRÉFAUt, MD
}

Objective To assess the effect of a training protocol on aerobic and anaerobic fitness in children with asthma.

Study design Sixteen boys (mean age: 13 years; range: 10-16 years) with mild-to-moderate asthma participated in a rehabilitation program that included 6 weeks of individualized training on a cycle ergometer. Two groups were randomly formed: the control group ( $\mathrm{CG}, \mathrm{n}=7$ ) and the training group ( $\mathrm{TG}, \mathrm{n}=9$ ), which exercised at an intensity set at the heart rate corresponding to the ventilatory threshold, with 1-minute sprints against the maximal aerobic power (MAP) every 4 minutes. Session duration was 45 minutes, 3 sessions per week. Changes in maximal oxygen uptake $\left(\mathrm{VO}_{2} \mathrm{max}\right)$, MAP, short-term peak power (PP), and pulmonary function were assessed.

Results Two patients of the training group did not complete the study. Pulmonary function remained unchanged in both groups. Improvement in both aerobic and anaerobic fitness was significant only in the training group $(\mathrm{TG}$ vs $\mathrm{CG})$ : VO ${ }_{2} \mathrm{max}+18 \%$ $\pm 2.1 \%$ versus $+9 \% \pm 4.5 \%(P<.05), \mathrm{MAP}+32 \% \pm 5 \%$ versus $12 \% \pm 7 \%(P<.05), \mathrm{PP}+21 \% \pm 5.7 \%$ versus $+8.8 \% \pm 10 \%(P<.01)$.

Conclusion Exercise training with high-intensity bouts is well tolerated in children with mild-to-moderate asthma. When included in a global rehabilitation program, this type of training improves both aerobic and anaerobic fitness. Anaerobic activities should be considered in sports rehabilitation programs for children with asthma. (J Pediatr 2003;142:179-84)

E xercise limitation is often encountered in children with asthma. The primary factor is generally thought to be their relative hyperventilation during exercise, which leads to dyspnea and exercise-induced bronchospasm (EIB). Self-limitation of physical activities then occurs to further avoid these unpleasent feelings. ${ }^{1}$ In children with asthma, muscle deconditioning through hypoactivity is thought to explain their low level of physical fitness. ${ }^{2}$ Several controlled studies support this concept: (1) children with asthma with a normal forced expiratory volume at one second $\left(\mathrm{FEV}_{1}\right)$ have normal habitual physical activity and exercise performance, ${ }^{2}(2)$ maximal oxygen uptake $\left(\mathrm{VO}_{2} \max \right)$ is correlated with physical activity, ${ }^{3}$ and (3) physical fitness in children with asthma can be enhanced by reconditioning programs. ${ }^{4-6}$ In contrast, an impaired exercise capacity independent of hypoactivity has been demonstrated in adult ${ }^{7}$ and pediatric patients, ${ }^{8}$ probably because of overinflated lungs, leading to ventilatory or cardiovascular limitation. As a result, decreased exercise capacity is consistently reported in children with asthma who have basal bronchial obstruction. ${ }^{9}$

On the basis of pathophysiologic concepts, training programs for the aerobic reconditioning of children with asthma ${ }^{10}$ have shown well documented benefits: the mean increase in maximal oxygen consumption normalized by body mass is approximately $15 \%,{ }^{4-6,11,12}$ with the anaerobic threshold shifted toward higher metabolic levels. ${ }^{5}$ Until recently, exercise capacity in asthmatic subjects has been studied only in terms of aerobic fitness. Yet it seems that the anaerobic component of exercise performance may also be important, because maximal anaerobic and maximal aerobic work capacities are decreased in

\begin{tabular}{llll}
\hline AT & Anaerobic threshold & MET & Metabolic equivalent \\
CG & Control group & PFT & Pulmonary function test \\
EIB & Exercise-induced brochospasm & PP & Peak power \\
FEV, & Forced expiratory volume at one second & $\mathrm{TG}$ & Training group \\
FV & Force-velocity test & $\mathrm{VO}_{2}$ & Oxygen consumption \\
LBM & Lean body mass & $\mathrm{VO}_{2}$ max & Maximal oxygen uptake \\
LLV & Lower limb volume & $\mathrm{VR}$ & Ventilatory reserve \\
MAP & Maximal aerobic power & & \\
\hline
\end{tabular}

From Service de Pédiatrie I, Service d'Exploration Fonctionnelle Respiratoire, Hôpital Arnaud de Villeneuve, and UFR STAPS, Laboratoire Sport Performance Santé, Montpellier, France.

Submitted for publication Feb 19, 2002; revisions received July 17, 2002, and Nov 15, 2002; accepted Nov 30, 2002

Reprint requests: F-P Counil, MD, PhD, Service de Pédiatrie I, 37| Avenue du Doyen Gaston Giraud, 34295 Montpellier, Cedex 5, France. E-mail: fcounil@chu-montpellier.fr.

Copyright @ 2003, Mosby, Inc. All rights reserved.

0022-3476/2003/\$30.00+0

10.1067/mpd.2003.83 


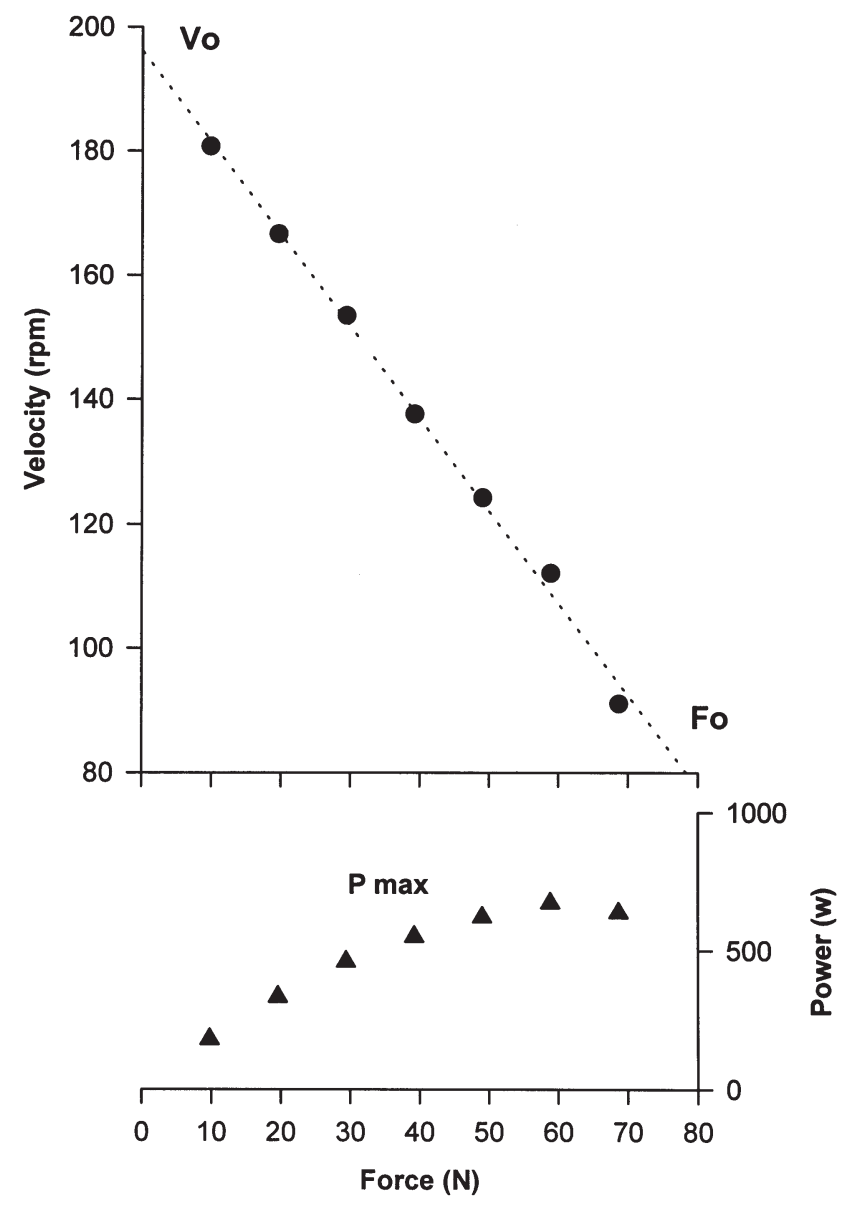

Figure. Example of typical force-velocity and force-power relationships during sprints on a cycloergometer (subject 3 ).

the same proportion in these patients. ${ }^{13}$ To ensure the overall satisfying physiologic development of the child with asthma, the need to restore both aerobic and anaerobic fitness appears to be a valid goal. To our knowledge, a training effect on the anaerobic component has not been demonstrated in patients with asthma, and training protocols are specifically designed to improve aerobic fitness. In healthy young adults, high-intensity training has a proven beneficial effect on both the aerobic and anaerobic capacity. ${ }^{14}$ It is thus logical to hypothesize that a combined aero-anaerobic training program would improve overall exercise performance in children with asthma. This study was therefore conducted to evaluate both aerobic and anaerobic conditioning after exercise training in asthmatic children with asthma.

\section{METHODS}

\section{Subjects}

Sixteen male children with a mean age of 13 years (range, 10-16 years) volunteered to participate in a rehabilitation program for subjects with asthma. These patients were attending two inpatient pulmonary rehabilitation clinics in Font Romeu, a small city in the Pyrenees Mountains of southwestern France. Inclusion in the study required one month of
Table I. Demographic data for 14 patients

\begin{tabular}{lcc}
\hline & $\begin{array}{c}\text { Control } \\
\text { group } \\
(\mathbf{n}=\mathbf{7})\end{array}$ & $\begin{array}{c}\text { Training } \\
\text { group } \\
(\mathbf{n}=\mathbf{7})\end{array}$ \\
\hline Mean age $(\mathrm{y})$ & $13.9 \pm 0.8$ & $14 \pm 0.6$ \\
Body mass $(\mathrm{kg})$ & $46.8 \pm 3.7$ & $48.9 \pm 3.2$ \\
Height $(\mathrm{cm})$ & $159.9 \pm 5.8$ & $159.4 \pm 2.9$ \\
Lean body mass $(\mathrm{kg})$ & $42 \pm 3.4$ & $42.1 \pm 2.3$ \\
Lower limb volume $(\mathrm{L})$ & $8.52 \pm 0.7$ & $9.45 \pm 0.6$ \\
Sexual maturation stages & $3(2-4)$ & $3(2-4)$ \\
IgE (kUI/L) & $1008.1 \pm 235$ & $944.7 \pm 197$ \\
MET & $6896 \pm 627$ & $6844 \pm 607$ \\
Inhaled corticosteroids & $5 / 7$ & $4 / 7$ \\
\hline
\end{tabular}

$M E T$, Metabolic equivalent for weekly physical activity.

acclimatization to altitude $(1400 \mathrm{~m}), 6$ weeks without any acute episode of wheezing, one year without emergency department visits or hospitalization for acute asthma, and a basal $\mathrm{FEV}_{1}>70 \%$ of predicted. The diagnosis of asthma was made on the basis of the following criteria: (1) personal or familial history of allergy; (2) personal history of acute wheezing; (3) reversible airway obstruction documented by lung function testing, ie, improvement of $15 \%$, at least in $\mathrm{FEV}_{1}$ and/or $30 \%$ in forced expiratory flow $25-75$ by inhaling a bronchodilatator; (4) positive specific immunoglobulin $\mathrm{E}$ to inhaled allergens by a multi-allergen allergosorbent test (Phadiatop, Pharmacia, Uppsala, Sweden) and/or a cutaneous hypersensitivity to one or several allergens; and (5) no evidence of other lung disease. A control group of 7 subjects was randomly formed from the 16 volunteers. Two patients of the training group did not complete the study. There were no significant differences in anthropometric characteristics, basal spirometry, and the habitual level of physical activity between the 2 groups (Table I). Nine of the 14 children were taking inhaled steroids.

Informed consent was obtained from the children and their parents before participation in the study; the protocol was approved by our local ethics committee.

\section{Measurements}

Lean body mass (LBM) was estimated from skinfold thickness measurements using the Durnin and Rahaman formula. ${ }^{15}$ Sexual maturity was scored with the Tanner indices. ${ }^{16}$ Lower limb volume (LLV) was evaluated according to the method of Jones and Pearson. ${ }^{17}$ Weekly physical activity estimates were based on a standardized activity interview ${ }^{18}$ and expressed in metabolic equivalents (MET). All children underwent pulmonary function testing (System 2800 Autobox, Sensormedics, Anaheim, Calif) at rest, and values were expressed as a percentage of pediatric standards. ${ }^{19}$

Aerobic fitness was first assessed by a maximal incremental exercise test on a cycle ergometer (864, Monark-Crescent AB, Varberg, Sweden). A 3-minute warm-up period at 


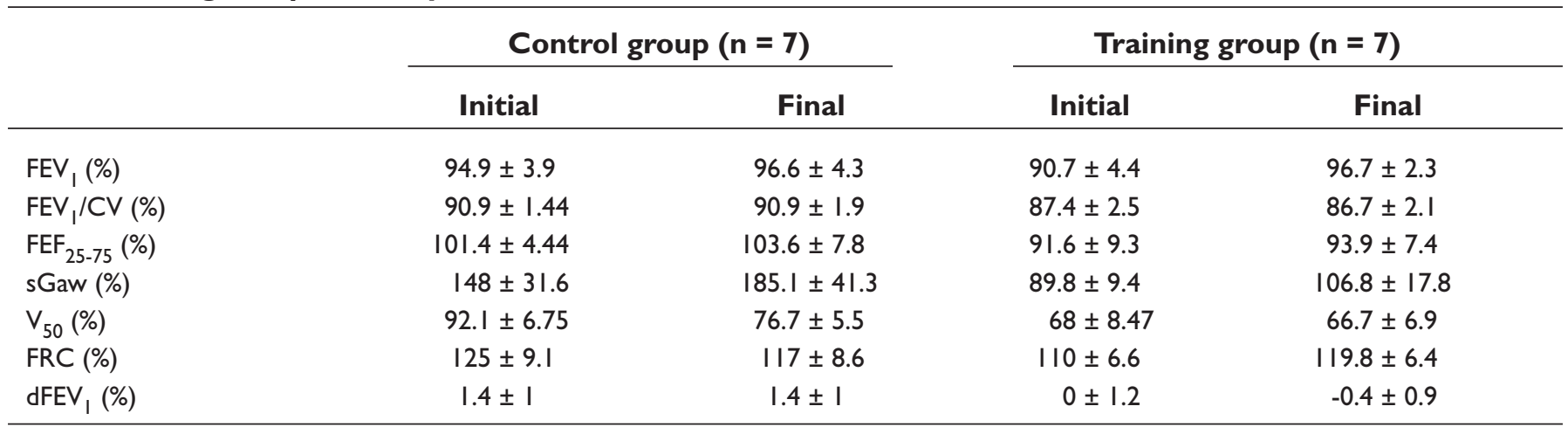

$V C$, Vital capacity; $F E F_{25-75}$, forced expiratory flow between $25 \%$ and $75 \%$ of vital capacity; $V_{50}$, expiratory flow at $50 \%$ of vital capacity; $s G a w$, specific conductance; $F R C$, functional residual capacity; $d F E V_{1}$, change in $\mathrm{FEV}_{1} 15$ minutes after the incremental exercise test.

$30 \mathrm{~W}$ was followed by $15-$ or $30-\mathrm{W}$ increments at 1 -minute intervals while the subject pedaled at a constant frequency of $60 \mathrm{rpm}$. Ventilation and gas exchange were measured with a breath-by-breath automated open circuit system (CPX, Medical Graphics, St Paul, Minn), for the calculation of oxygen consumption $\left(\mathrm{VO}_{2}\right)$. Exercise continued until at least 3 of the maximal oxygen uptake criteria were obtained ${ }^{20}:(1)$ a plateau of $\mathrm{VO}_{2}$ in spite of the increase of workload, (2) maximal heart rate (maximal predicted heart rate $\pm 5 \%$ ), (3) respiratory exchange ratio $>1.10$, and (4) inability to maintain the pedaling frequency at $60 \mathrm{rpm}$. The maximal aerobic power (MAP) reached during the test was noted. The anaerobic threshold (AT) was the disproportionate increase in carbon dioxide output measured by the method of Beaver et al. ${ }^{21}$ The corresponding heart rate, $\mathrm{VO}_{2}$, and power were noted. The ventilatory reserve (VR) was expressed as percentage of predicted maximal minute ventilation:

$$
\left.\mathrm{VR}=100 \cdot\left(\left[\mathrm{FEV}_{1} \cdot 35\right]-\mathrm{VEmax}\right) /\left[\mathrm{FEV}_{1} \cdot 35\right]\right)
$$

Anaerobic performance was assessed by the force-velocity test $(\mathrm{FV})$. Briefly, the subject was asked to perform repeated maximal sprints against an increasing breaking load $(\mathrm{F})$ with a 5-minute recovery period between each sprint. ${ }^{22}$ The breaking load was increased by 0.5 or $1 \mathrm{~kg}$ according to body dimension. Sprint duration was fixed at 6 seconds, the maximum time it took for the vigorously motivated subject to attain his maximal pedaling frequency after the starting signal. ${ }^{23}$ The maximal pedaling frequency $(\mathrm{rpm})$ was recorded and the linear force-velocity and parabolic force-power relationships were calculated by an automated system ${ }^{24}$ that allowed determination of both $\mathrm{V}$ from the measurement of pedaling frequency and, for each $\mathrm{F}$, the power corresponding to the product $\mathrm{F} \cdot \mathrm{V}$. The accuracy of the pedal revolution duration was $3.3 \mathrm{~ms}$. The test was stopped when there was no longer any increase in the power output in spite of an increasing breaking load. The maximal anaerobic peak power (PP) was calculated from the parabolic force-power relationship. The intercepts of the FV regression line determined Fo and Vo. Fo is the maximal breaking mass the subject could theoritically handle; Vo is the maximal cycling velocity theoretically obtained if the breaking mass was null (Figure).

\section{Training Programs}

The training group exercised 3 times weekly for 6 weeks, with each session consisting of 45 min0utes of continuous cycling activity. The design of the training sessions was adapted from Gimenez et al. ${ }^{25}$ The target heart rate was individualized and corresponded to the AT level. Every 4 minutes, the subject was asked to sprint for 1 minute against the breaking load corresponding to his MAP. During all sessions, heart rate was continuously monitored with a cardiofrequency meter (Sport Tester PE 3000, Polar Electro, Kemple, Finland). A training instructor and a pulmonologist supervised each session to ensure that the clinical condition was stable and the training procedures were followed.

\section{Protocol}

All subjects underwent clinical examination, anthropometric measurements, ECG and spirometry before entering in the study. They then familiarized themselves with the exercise testing procedures. Each subject performed one incremental exercise test and one FV test, with pre- and postexercise pulmonary function testing. The same evaluation was repeated when the 6 weeks of training were completed. Testing was done blindly regarding the training groups. Peak flow rates were monitored twice daily. All treatment was given under direct medical supervision. Asthma exacerbations with oral corticosteroid use and hospitalization for any reason during the protocol were exclusion criteria.

\section{Statistical Analysis}

Means \pm SD values are reported. A Mann-Whitney Wilcoxon rank test was used for between-group comparisons. A 2-way analysis of variance was conducted to evaluate the group $\times$ time effect on pulmonary function and fitness parameters. Multiple regression models were applied to assess the relative contribution of the independent variables to the total variance of the fitness variables $\left(\mathrm{VO}_{2} \max , \mathrm{MAP}, \mathrm{PP}\right)$. These potentially confounding variables were anthropometric 


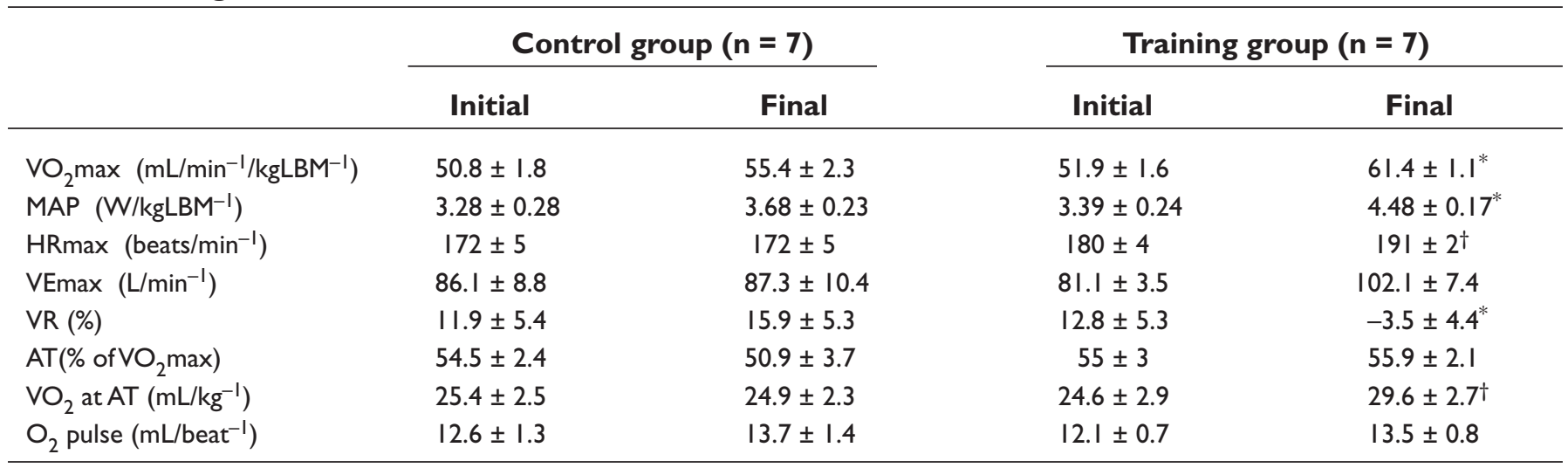

$V \mathrm{O}_{2}$ max, maximal oxygen uptake; Pmax, maximal power during $\mathrm{VO}_{2}$ max testing; HRmax, maximal heart rate; $A T$, anaerobic threshold; VEmax, maximal minute ventilation; $V R$, ventilatory reserve.

Values are means with SD.

${ }^{*} P<.05$.

$\dagger P<.01$.

\section{Table IV. Changes in anaerobic fitness}

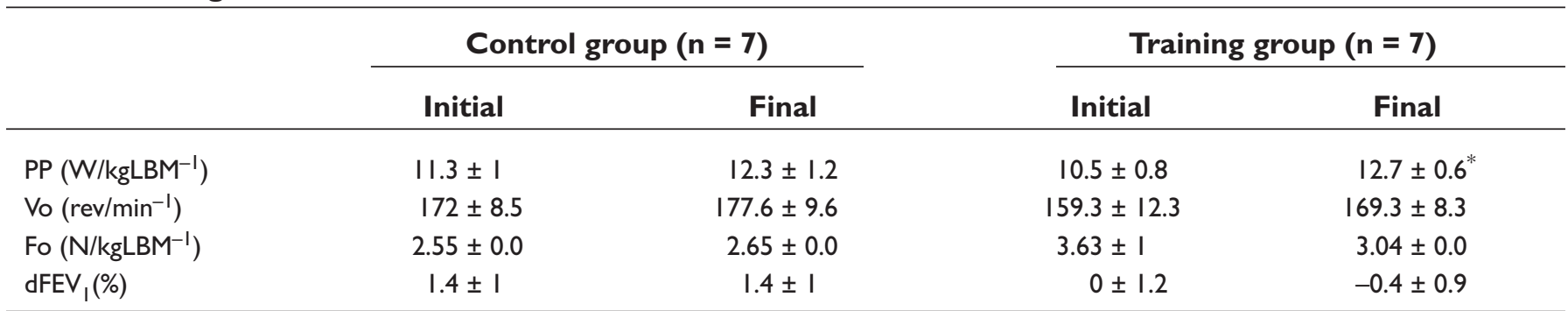

$P P$, Peak power reached during the force-velocity test; $F_{0}$ and $V o$, intercepts of the linear force-velocity relationship. $d F E V_{1}$, change in FEV 15 minutes after the force-velocity test.

${ }^{*} P<.01$.

data, sexual maturation, pulmonary function tests (PFTs), initial versus final evaluation (learning effect) and training. The level of statistical significance was set at $P<.05$.

\section{RESULTS}

\section{Tolerance and Side Effects}

The exercise tests were well tolerated. Peak power performed during the FV test was 3-fold higher than MAP. During the training sessions, the maximal drop in peak flow was $-40 \%$. Two subjects did not complete the training program because of a limb fracture unrelated to training in one and fatigue and lack of motivation in the other. The basal PFTs were not modified in these 2 patients and their clinical course remained stable. PFTs and lability of postexercise airways flows were not modified in either group (Table II).

\section{Aerobic Fitness (Table III)}

No statistical difference was found at the initial evaluation. After 6 weeks, the training group exhibited a significant increase in $\mathrm{VO}_{2} \max (+18 \pm 2.1 \%$ vs $+9 \pm 4.5 \%, P<.05)$ and MAP $(+32 \pm 5 \%$ vs $12 \pm 7 \%, \mathrm{P}<.05)$, whereas no significant changes were observed in the control group. Maximal heart rate was increased and ventilatory reserve was decreased after training only. $\mathrm{VO}_{2}$ at $\mathrm{AT}$ was significantly higher post-training than pretraining. Multiple regression models applied to the whole population $(n=14)$ revealed that the best subsets model explaining the variance of $\mathrm{VO}_{2}$ max and MAP were a linear function of training $\left(r^{2}=\right.$ $0.4, P=.0003)$ and a linear combination of sexual maturation and training $\left(r^{2}=0.53, P=0.006\right)$, respectively. Anthropometric data, daily activity, initial versus final $\mathrm{VO}_{2} \max$ or MAP measurements, and PFTs did not significantly improve the models when added.

\section{Anaerobic Fitness (Table IV)}

Initial evaluation did not reveal any significant difference between the 2 groups for Vo, Fo, and PP, although initial Vo seemed to be lower in the training group $(P=.4)$. After 6 weeks, the trained group exhibited an improved PP, whereas the control group remained stable $(+8.8 \pm 9.7 \%$ vs $+21 \pm 6.7 \%$, $P<.01)$. Multiple regression models applied to the whole population $(\mathrm{n}=14)$ revealed that the best subsets regression explaining the variance of $\mathrm{PP}$ was a linear combination of height, daily activity, and initial versus final PP measurements $\left(r^{2}=0.69, P=.0002\right)$. Other anthropometric data, training, sexual maturation, and PFTs did not significantly improve the regression models when added. 


\section{DISCUSSION}

The anaerobic fitness of asthmatic children can be improved by a specific training protocol that includes a combination of ventilatory threshold workloads and highintensity workloads. This protocol was well tolerated by children with stabilized, mild-to-moderate asthma, which was shown by the absence of significant adverse reactions. One subject of the 7 who completed the training protocol, however, had a poor tolerance attributed to either muscular fatigue or poor motivation. As part of a rehabilitation protocol, a combined aero-anaerobic training program gave specific and significant benefits in terms of physical fitness to those who completed it.

The accurate and reliable evaluation of the effects of physical conditioning is generally difficult in children with asthma. Our children were within normal ranges for daily activity for French children. ${ }^{26}$ The most striking point is that children in training programs receive extra attention as they exercise under the supervision of health professionals and are thus in a privileged situation regarding monitoring of their disease. They may therefore benefit from more finely adjusted treatment leading to therapeutic compliance. For this study it was thus very important to ensure a similar level of medical attention to the control group. Our population, recruited from 2 long-stay clinics dedicated to the rehabilitation of asthmatic children, was ideal for this purpose. The daily monitoring of clinical status, physical activity, peak expiratory flow, and therapeutic compliance involved all patients and ensured the optimal care of the control group as well. It is likely that this situation explains the remarkably stable condition of all patients throughout the study. The absence of significant changes in the daily treatment and the PFTs confirmed the clinical stability of the entire population and excluded variability in pulmonary function as an influence on the physical fitness evaluation.

Another major concern regarding the evaluation of pediatric exercise capacity is the child's commitment during testing. Although specific pediatric criteria for aerobic fitness evaluation are lacking, the peak $\mathrm{VO}_{2}$ measured during an incremental exercise test has been reported to be a valid tool for $\mathrm{VO}_{2}$ max determination in children. ${ }^{27}$ In our study, the initial $\mathrm{VO}_{2}$ max and MAP values were within the range of healthy active boys of similar age, ${ }^{28}$ indicating good participation. Measurement of blood acidosis would have been of interest to further confirm the criteria for maximal exercise levels, but we considered this procedure too invasive. Our strongest indication, in fact, of a valid evaluation of aerobic fitness was the finding that all children in both groups met the criteria we set for $\mathrm{VO}_{2}$ max determination, especially a respiratory exchange ratio above 1.1. During the FV test, it was easier to verify that maximal power was reached. As stated above, the relationship between force and velocity is quasilinear. Submaximal cycling would have led to aberrant points, which was not the case for any of the subjects tested. All of the PP values observed in our population were in the range of normal pediatric values. $^{29}$
The improvement in aerobic fitness confirmed previous reported findings in children with asthma. ${ }^{4,5}$ Only a few controlled studies are available on the effects of endurance training in children with asthma. ${ }^{4-6,11,12}$ Although $\mathrm{VO}_{2} \max ^{4,5,11}$ and $\mathrm{MAP}^{4,11}$ have shown improvement, and AT shifted toward a higher metabolic level ${ }^{5}$ in some studies, no significant changes in $\mathrm{VO}_{2}$ max, ${ }^{6,12} \mathrm{MAP}, 6,12$ or $\mathrm{AT}^{6}$ were observed in others. In our study, the trained children with asthma increased their maximum heart rate and used more of their ventilatory reserve, which suggests improved tolerance to exercise. They shifted their AT toward a higher metabolic level, and training was the main explanatory factor of the variation in the total variance of their $\mathrm{VO}_{2} \max$ and MAP, suggesting a true aerobic training effect. It is very likely that this improvement can be attributed to muscular conditioning rather than cardiorespiratory adaptation since the PFTs and the $\mathrm{O}_{2}$ pulse remained stable after training: the theoretic maximal ventilatory reserve (35 $\mathrm{FEV}_{1}$ ) and the maximal stroke volume as evaluated by the $\mathrm{O}_{2}$ pulse were not significantly modified by the training protocol.

Current data have shown that supramaximal power ouputs are generally diminished whatever the type of exercise done by children with mild-to-moderate asthma. Anaerobic fitness as evaluated by the Wingate test, a 30second all-out cycling test, has been described as either nor$\mathrm{mal}^{30}$ or decreased ${ }^{13}$ when compared with that of healthy controls. Interestingly, patients with significant basal bronchial obstruction, ie, patients with cystic fibrosis ${ }^{30}$ or moderate asthma, ${ }^{13}$ have shown diminished maximal power output during the Wingate test. Such a test, however, gives a global picture of aerobic and anaerobic fitness, because the contribution of the aerobic pathway to total energy production is significant in children. ${ }^{31}$ Shorter exercise bouts like those of the FV test are mainly anaerobic and glycolytic, ${ }^{32,33}$ and the anaerobic fitness evaluated by this test was also found to be diminished in children with asthma. ${ }^{34} \mathrm{In}$ our training group, the increase in PP seems related more to an increase in Vo, suggesting a training effect on velocity. Training, however, was a weak explanatory factor of the variation in the total variance of $\mathrm{PP}$, and confounding effects of anthropometry or daily activity cannot be ruled out. The effectiveness of anaerobic training would have probably been more pronounced with supramaximal training loads, ${ }^{35}$ but the risks and benefits of strength training for children have not yet been extensively studied.

In conclusion, aerobic and anaerobic exercise fitness can both be enhanced by specific training in children with mild-to-moderate asthma. This type of training is well tolerated. When bronchial obstruction is alleviated, the mechanism of muscle conditioning is probably not very different from that of healthy children. Further controlled studies are nevertheless needed to determine whether atopic asthma is associated with a specific pattern of muscle fiber conditioning. Organized sports activities should include short and intense bouts of muscle work and should be proposed to stable asthmatic children. 


\section{REFERENCES}

1. Préfaut C, Varray A, Vallet G. Pathophysiological basis of exercise training in patients with chronic obstructive lung disease. Eur Respir Rev 1995;5:27-32.

2. Santuz P, Baraldi E, Filipone M, Zachello F. Exercise performance in children with asthma: is it different from that of healthy controls? Eur Respir J 1997;10:1254-60.

3. Garfinkel SK, Kesten S, Chapman KR, Rebuck AS. Physiologic and nonphysiologic determinants of aerobic fitness in mild to moderate asthma. Am Rev Respir Dis 1992;145:741-5.

4. Orenstein DM, Reed ME, Grogan FT, Crawford LV. Exercise conditioning in children with asthma. J Pediatr 1985;106:556-60.

5. Varray AL, Mercier JG, Terral CM, Prefaut CG. Individualized aerobic and high intensity training for asthmatic children in an exercise readaptation program. Chest 1991;99:579-86.

6. Neder JA, Nery LE, Silva AC, Cabral ALB, Fernandes ALG. Short term effect of aerobic training in the clinical management of moderate to severe asthma in children. Thorax 1999;54:202-6.

7. Baab TG, Long KA, Rodarte JR. The relationship between maximal expiratory flow and increases of maximal exercise capacity with exercise training. Am J Respir Crit Care Med 1997;156:116-21.

8. Varray A, Mercier J, Savy-Pacaux AM, Prefaut C. Cardiac role in exercise limitation in asthmatic subjects with special reference to disease severity. Eur Respir J 1993;6:1011-7.

9. Varray A, Prefaut C. Physiopathological bases for retraining programs of asthma patients: adjustment to rehabilitation. Rev Mal Respir 1992;9:355-66.

10. Varray A, Prefaut C. Exercise training in patients with respiratory disease: procedures and results. Eur Respir Rev 1995;5:51-8.

11. Ahmaidi SB, Varray AL, Savy-Pacaux AM, Prefaut CG. Cardiorespiratory fitness evaluation by the shuttle test in asthmatic subjects during aerobic training. Chest 1993;103:1135-41.

12. Matsumoto I, Araki H, Tsuda K, Odajima H, Nishima S, Higaki Y, et al. Effect of swimming training on aerobic capacity and exercise-induced bronchoconstriction in children with bronchial asthma. Thorax 1999;54:196-201.

13. Counil F-P, Varray A, Karila C, Hayot M, Voisin M, Prefaut C. Wingate test performance in children with asthma: aerobic or anaerobic limitation? Med Sci Sports Exerc 1997;29:430-5.

14. Tabata I, Nishimura K, Kouzaki M, Hirai Y, Ogita F, Miyachi M, et al. Effects of moderate-intensity endurance and high-intensity intermittent training on anaerobic capacity and $\mathrm{VO}_{2} \max$. Med Sci Sports Med 1996;28:1327-30.

15. Durnin J, Rahaman M. The assessment of the amount of fat in human body from measurements of skinfold thickness. Br J Nutr 1967;21:681-9.

16. Tanner JM. Physique and body composition. In: Larson LA, editor. Fitness, health, and work capacity. New York: Macmillan; 1974. p. 516-51.

17. Jones PRM, Pearson J. Anthropometric determination of leg fat and muscle plus bone volume in young male and female adults. J Physiol 1969;204:63-6.

18. Verschuur R, Kemper HCG. Habitual physical activity. Med Sport Sci 1985;20:56-65.
19. Zappletal A, Samanek M, Paul T. Lung function in children and adolescents. In: Herzog H, editor. Progress in respiration research. Farmington (CT): Karger Press; 1987. p. 113-218.

20. Spiro SG. Exercise testing in clinical medicine. Br J Dis Chest 1977;71:145-72.

21. Beaver WL, Wasserman K, Whipp BJ. A new method for detecting anaerobic threshold by gas exchange. J Appl Physiol 1986;60:2020-7.

22. Vandewalle H, Peres G, Heller J, Panel J, Monod H. Force-velocity relationships and maximal power on a cycle ergometer. Eur J Appl Physiol 1987;56:650-6

23. Mercier B, Mercier J, Granier P, Le Gallais D, Préfaut Ch. Maximal anaerobic power: relationship to anthropometric characteristics during growth. Int J Sports Med 1992;13:21-6.

24. Mercier J, Mercier B, Prefaut Ch. Blood lactate during the force-velocity exercise test. Int J Sports Med 1991;12:17-20.

25. Gimenez M, Serrera E, Salinas W. Square wave endurance exercise test (SWEET) for training and assessment in trained and untrained subjects. I. Description and cardiorespiratory responses. Eur J Appl Physiol 1982; 49:359-68.

26. Counil FP, Karila C, Varray A, Guillaumont S, Voisin M, Prefaut C. Anaerobic fitness in children with asthma: adaptation to maximal intermittent short exercise. Pediatr Pulmonol 2001;31:198-204.

27. Armstrong $\mathrm{N}$, Welsman J, Winsley R. Does peak $\mathrm{VO}_{2}$ reflect $\mathrm{VO}_{2} \max$ in children? Evidence from supra-maximal testing. Int J Sports Med 1995; 17:356-9.

28. Ekelund U, Poortvliet E, Nilsson A, Yngve A, Holmberg A, Sjostrom M. Physical activity in relation to aerobic fitness and body fat in 14- to 15year-old boys and girls. Eur J Appl Physiol 2001;85:195-201.

29. Van Praagh E, Fellmann N, Bedu M, Falgairette G, Coudert J. Gender difference in the relationship of anaerobic power output to body composition in children. Pediatr Exerc Sci 1990;2:336-48.

30. Boas SR, Danduran MJ, McColley SA. Energy metabolism during anaerobic exercise in children with cystic fibrosis and asthma. Med Sci Sports Exerc 1999;31:1242-9.

31. Naughton GA, Carlson JS, Buttifant DC, Selig SE, Meldrum K, McKenna MJ, et al. Accumulated oxygen deficit measurements during and after high-intensity exercise in trained male and female adolescents. Eur J Appl Physiol Occup Physiol 1997;76:525-31.

32. Serresse O, Lortie G, Bouchard C, Boulay MR. Estimation of the contribution of the various energy systems during maximal work of short duration. Int J Sports Med 1988;9:456-60.

33. Sargeant AJ. The determinants of anaerobic muscle function during growth. In: Van Praagh E, editor. Pediatric anaerobic performance. Champaign (IL): Human Kinetics; 1998. p. 97-117.

34. Counil FP, Karila C, Varray A, Guillaumont S, Voisin M, Prefaut C. Anaerobic fitness in children with asthma: adaptation to maximal intermittent short exercise. Pediatr Pulmonol 2001;31:198-204.

35. Blimkie CJR, Sale DG. Strength development and trainability during chilhood. In: Van Praagh E, editor. Pediatric anaerobic performance. Champaign (IL): Human Kinetics; 1998. p. 193-224. 